\title{
Resolving inflammation in nonalcoholic steatohepatitis
}

\begin{abstract}
Matthew Spite
Center for Experimental Therapeutics and Reperfusion Injury, Department of Anesthesiology, Perioperative and Pain Medicine, Brigham and Women's Hospital and Harvard Medical School, Boston, Massachusetts, USA.
\end{abstract}

\begin{abstract}
Chronic unresolved inflammation contributes to the development of nonalcoholic steatohepatitis (NASH), a disorder characterized by lipotoxicity, fibrosis, and progressive liver dysfunction. In this issue of the $J C I$, Han et al. report that maresin 1 (MaR1), a proresolving lipid mediator, mitigates NASH by reprograming macrophages to an antiinflammatory phenotype. Mechanistically, they identified retinoic acid-related orphan receptor $\alpha(\mathrm{ROR} \alpha)$ as both a target and autocrine regulator of MaR1 production. Because NASH is associated with many widely occurring metabolic diseases, including obesity and type 2 diabetes, identification of this endogenous protective pathway could have broad therapeutic implications.
\end{abstract}

\section{Inflammation and its} resolution: role of maresins

Acute inflammation is a temporally coordinated host response to invading pathogens and tissue injury. Instructed by endogenous mediators, distinct leukocyte subsets sequentially follow defined routes, carry out specialized tasks, and ultimately clear from the tissue so that its function can be restored. Proinflammatory mediators, such as chemokines and lipid mediators (e.g., leukotrienes, prostaglandins), promote vascular permeability, amplify leukocyte infiltration, and stimulate the release of substances that assist in pathogen eradication. Resolution of inflammation begins with the biosynthesis of mediators that terminate neutrophil infiltration and proinflammatory mediator production to prevent excessive damage to healthy tissue. These specialized proresolving mediators (SPMs), such as the maresins, also stimulate phagocytes to clear dead cells and bacteria, and they actively engage tissue reparative programs (1). Thus, SPMs are host-protective mediators that temper inflammation without causing immunosuppression.
Maresins (macrophage mediators in resolving inflammation) were originally discovered by Serhan et al. in resolving inflammatory exudates as novel proresolving molecules (2). Their complete structures with stereochemical assignments have been systematically elucidated, and the mechanisms of their biosynthesis have been defined. Maresin 1 (MaR1) is biosynthesized from docosahexaenoic acid (DHA) via 12-lipoxygenase-mediated (12-LOXmediated) production of a 14-hydroperoxy intermediate that undergoes enzymatic epoxidation to form $13 S, 14 S$-epoxy maresin (eMaR) $(2,3)$. This epoxide is enzymatically converted to MaR1 ( $7 R, 14 S$-dihydroxy-4Z,8E,10E,12Z,16Z,19Z-docosahexaenoic acid) and MaR2, or can be conjugated with glutathione to produce the newly characterized cysteinyl SPM (2-5). Maresins blunt neutrophil chemotaxis and promote macrophage efferocytosis in animal models of acute inflammation. They have direct actions on human macrophages, are organ protective, and stimulate tissue regeneration $(2,6,7)$. Earlier studies have also determined that MaR1, as well

Related Article: p. 1684

Conflict of interest: The author has declared that no conflict of interest exists.

as other SPMs such as resolvins and protectins, resolve chronic inflammation and improve systemic metabolism and hepatic steatosis in mouse models of obesity and type 2 diabetes (8-11). In this issue, Han et al. add new insights into the regulation of MaR1 biosynthesis in obesity and identify downstream pathways engaged by MaR1 in macrophages that could, in part, underlie its protective actions (12).

Nonresolving inflammation in nonalcoholic steatohepatitis

While acute inflammation normally resolves, accumulating evidence indicates that maladaptive unresolved inflammation contributes to several chronic diseases, including nonalcoholic steatohepatitis (NASH) (13). The development of NASH is associated with diseases of altered systemic metabolism, such as type 2 diabetes, obesity, and hyperlipidemia. Lipotoxicity in hepatocytes occurs as a result of an imbalance between lipogenesis, secretion, and fatty acid $\beta$-oxidation, and can lead to apoptosis and necrosis. Release of endogenous damage-associated molecular patterns (DAMPs) initiates a sterile inflammatory response characterized by robust proinflammatory cytokine production and accumulation of leukocytes (e.g., neutrophils, macrophages, dendritic cells, or Th17 cells). Macrophages polarized to a proinflammatory state play a critical role in the progression of NASH, both in perpetuating chronic inflammatory signaling via the production of inflammatory cytokines (e.g., TNF- $\alpha$ and IL-1 $\beta$ ) and in promoting fibrosis via the release of profibrotic cytokines (i.e., TGF- $\beta 1$ ) (13). In contrast, polarization of liver macrophages to an antiinflammatory (i.e., M2) phenotype protects against the progression of NASH.

Previous studies determined a critical role of the nuclear receptor retinoic acid-related orphan receptor $\alpha(\operatorname{ROR} \alpha)$ in the regulation of lipid metabolism and inflammation in NASH (14-16). In a pre- 


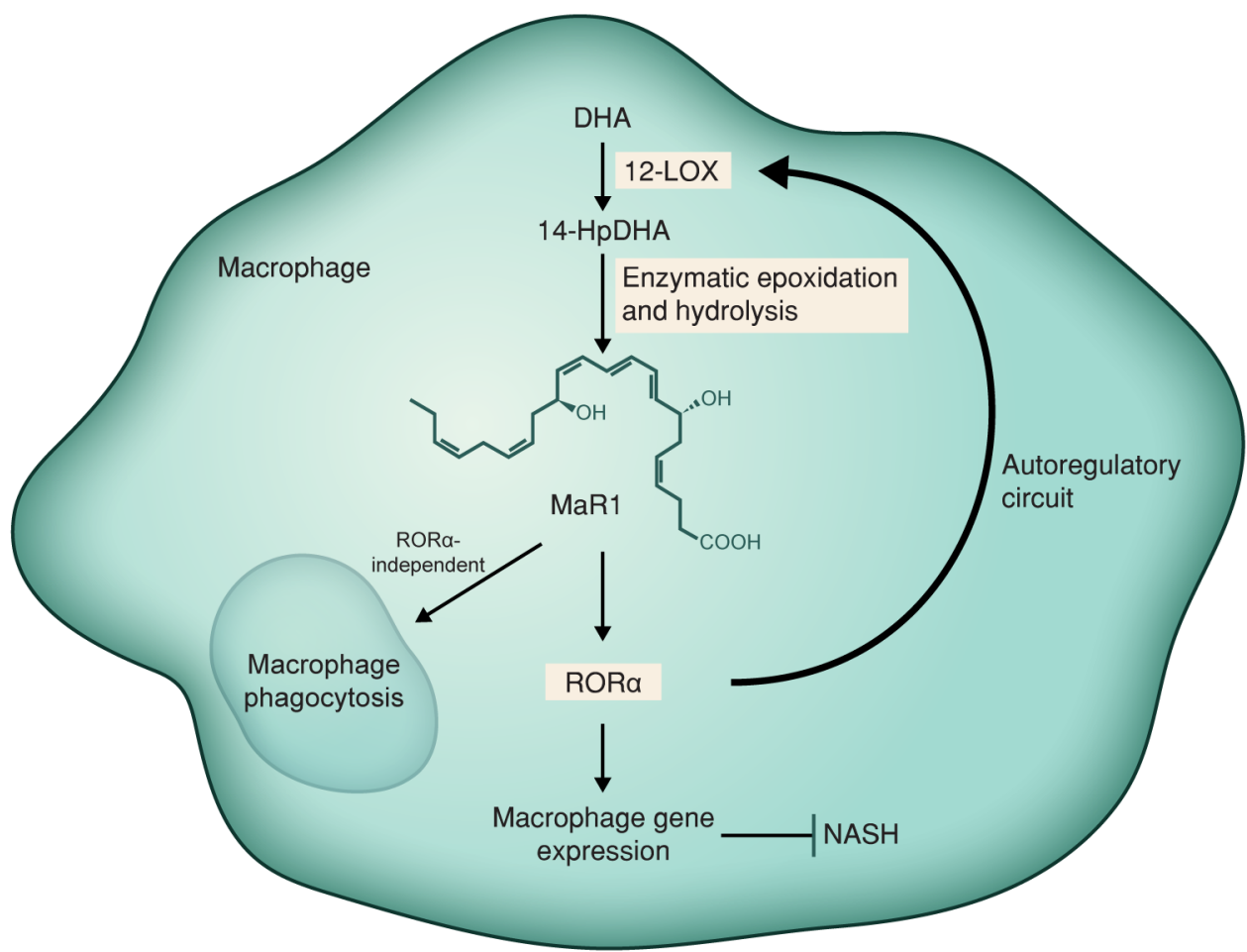

Figure 1. In macrophages, 12-LOX converts DHA to a 14-hydroperoxy DHA (hpDHA) intermediate that undergoes enzymatic epoxidation and hydrolysis to form MaR1. Han et al. found that MaR1 binds to and activates ROR $\alpha$, which leads to an antiinflammatory macrophage phenotype that blunts the progression of NASH. In addition, autoregulation of 12-LOX expression by ROR $\alpha$ establishes a positive feed-forward circuit. Biological actions of MaR1 that are key during resolution of inflammation, such as macrophage phagocytosis, were found to be independent of ROR $\alpha$. Illustration by Rachel Davidowitz. vious study, Han et al. reported that $\mathrm{ROR} \alpha$ is important for polarizing liver macrophages to an antiinflammatory phenotype during NASH (17). In their present study, Han et al. hypothesized that, because of the interplay between $\mathrm{ROR} \alpha$ and lipid metabolism and inflammation in the liver, lipid-derived mediators may regulate $\mathrm{ROR} \alpha$ activation for resolution. Since DHA has been shown to improve NASH and chronic inflammation in several other contexts (11), they questioned whether ROR $\alpha$ contributes to these protective actions. In initial studies, they found that DHA increased expression of ROR $\alpha$ and that this was associated with increased M2 macrophage polarization. Similar results were obtained in fat-1 transgenic mice, which have high endogenous levels of DHA in the liver. Interestingly, the regulation of macrophage polarization by DHA was lost in macrophages from mice with myeloid-specific deficiency of ROR $\alpha$ (ROR $\alpha$-MKO), establishing that activation of ROR $\alpha$ mediates, in part, the role of DHA in this context.

\section{MaR1: a novel agonist} for ROR $\alpha$ ?

Earlier studies reported that DHA activates retinoid $\mathrm{X}$ receptor (18). Han et al. questioned whether DHA is also a ligand for ROR $\alpha$. While DHA increased a ROR $\alpha$ reporter gene, DHA itself was not a direct ligand for ROR $\alpha$ based on failure to recruit a coactivator peptide as compared with a synthetic ROR $\alpha$ agonist. Because DHA is known to be converted in macrophages to bioactive lipid mediators that resolve inflammation (1), the authors tested whether specific SPMs regulated ROR $\alpha$ in macrophages. From these studies, the authors determined that MaR1, but not other SPMs (i.e., resolvin D1), increased expression of $\mathrm{ROR} \alpha$ coincident with an increase in M2 macrophage polarization, which was both time- and dose-dependent. Of note, these results are consistent with prior studies showing that MaR1 regulates a proresolving macrophage phenotype (3). The regulation of M2 polarization was abolished by genetic deficiency of Rora. Consistent with activation of $\mathrm{ROR} \alpha, \mathrm{MaR} 1$ promoted binding of coactivator p300 concomitant with decreased binding of NCoR1, a corepressor of ROR $\alpha$. Structure function analysis demonstrated that several other closely related SPMs (e.g., RvD1, RvD2, and protectin D1) did not modulate ROR $\alpha$ and that activation of ROR $\alpha$ by MaR1 was specific, as other nuclear receptors including ROR $\beta$, $\mathrm{ROR} \gamma$, retinoid $\mathrm{X}$ receptor $\alpha$, peroxisome proliferator-activated receptor $\gamma$, and liver $\mathrm{x}$ receptor $\alpha$, were not activated by MaR1.
Like DHA and consistent with prior studies, treatment of obese mice with MaR1 prevented the development of NASH, as evidenced by reduced liver weight, hepatic triglyceride levels, markers of liver injury, and expression of profibrotic proteins. Similarly, MaR1 treatment increased the appearance of $\mathrm{M} 2$ polarized macrophages in the liver of obese mice. Notably, these protective effects were lost in ROR $\alpha$-MKO mice, establishing causality in the activation of this pathway by MaR1 in vivo.

The activation of ROR $\alpha$ by MaR1 is surprising because most SPMs, as well as other lipid mediators, elicit their biological actions by binding to and activation of plasma membrane $G$ protein-coupled receptors, rather than nuclear receptors $(19,20)$. Nonetheless, using surface plasmon resonance, fluorescence resonance energy transfer assays, and molecular modeling, Han et al. systematically demonstrated that MaR1 is likely an agonist of $\mathrm{ROR} \alpha$. Transcriptome profiling of macrophages stimulated with MaR1 showed some overlap with genes induced by a synthetic ROR $\alpha$ agonist (i.e., SR1078), although it should be noted that MaR1 distinctly regulated a large subset of genes as well. In addition, MaR1 is known to be metabolized in macrophages to further downstream products (e.g., 14-oxoMaR1) 
(21). Whether these metabolites or MaR1 itself serve as the actual ligand(s) for $\mathrm{ROR} \alpha$ remains to be determined. Moreover, acute activation of macrophage phagocytosis by MaR1 was ROR $\alpha$-independent and occurred at much lower concentrations and shorter time periods than that needed to activate $\mathrm{ROR} \alpha(10 \mathrm{nM}$ vs. $>50 \mathrm{nM})$. Thus, given that stimulation of macrophage phagocytosis is a key biological function of MaR1 during resolution of acute inflammation $(1,2)$, these results suggest that an as-yet-unidentified receptor may distinctly mediate this process.

\section{Feed-forward loop for the activation of ROR $\alpha$}

As noted above, several chronic diseases such as NASH are associated with nonresolving inflammation. Given that the symptoms of NASH could be partially alleviated by MaR1, Han et al. next questioned whether its development is associated with an impairment in MaR1 biosynthesis. To this end, they established that the expression of key MaR1 biosynthetic enzyme ALOX12 (encoding 12-LOX) is inversely associated with NASH in humans. In mice, development of NASH was associated with decreased hepatic levels of MaR1. Expression of Alox12 was decreased in ROR $\alpha$ MKO mice, indicating that, in addition to serving as a target for MaR1, ROR $\alpha$ may also regulate the MaR1 biosynthetic machinery. To test this, the authors treated mice with SR1078 and found that MaR1 increased in WT mice. Similarly, treatment of mice with DHA increased MaR1 levels in WT mice but not in ROR $\alpha$-MKO mice. Further experiments determined that Alox12 is directly regulated by $\mathrm{ROR} \alpha$ binding and overexpression of Alox12 together with DHA treatment protected mice from NASH, whereas DHA was ineffective in mice treated with a 12-LOX inhibitor. Altogether, these results establish a feed-forward autoregulatory loop in which MaR1 activates $\mathrm{ROR} \alpha$ in macrophages, which in turn further increases transcription of Alox12 to potentiate MaR1 production (see Figure 1). It should be noted that MaR1 also has direct protective actions on hepatocytes, and thus local macrophage-derived MaR1 could potentially dampen steatosis via these additional cellular targets as well (22).

\section{Future directions}

In summary, the study by Han et al. adds new insight into the endogenous biosynthesis and actions of MaR1. Because ROR $\alpha$ plays numerous roles in cellular metabolism and inflammation, it will be interesting to determine the role of this pathway in regulating other protective actions of MaR1 (23). For example, it has been reported that mice deficient in Rora develop severe atherosclerosis, a progressive chronic inflammatory disease that is also improved by MaR1 $(24,25)$. Nonetheless, key biological roles of MaR1 during resolution, such as enhancement of macrophage phagocytosis, were found to be independent of ROR $\alpha$, suggesting that previously undescribed receptors are likely to also contribute to these well-characterized roles in vivo.

\section{Acknowledgments}

The author's laboratory is supported in part by NIH grants HL106173 and GM095467.

Address correspondence to: Matthew Spite, Hale Building for Transformative Medicine, 60 Fenwood Road, Room 3016K, Boston, Massachusetts 02115, USA. Phone: 617.525 . 5133; Email: mspite@bwh.harvard.edu.

1. Serhan CN, Levy BD. Resolvins in inflammation emergence of the pro-resolving superfamily of mediators. JClin Invest. 2018;128(7):2657-2669.

2. Serhan CN, et al. Maresins: novel macrophage mediators with potent antiinflammatory and proresolving actions. JExp Med. 2009;206(1):15-23.

3. Dalli J, et al. The novel 13S,14S-epoxy-maresin is converted by human macrophages to maresin 1 (MaR1), inhibits leukotriene A4 hydrolase (LTA4H), and shifts macrophage phenotype. FASEB J. 2013;27(7):2573-2583.

4. Dalli J, Chiang N, Serhan CN. Identification of 14-series sulfido-conjugated mediators that promote resolution of infection and organ protection. Proc Natl Acad Sci US A. 2014;111(44):E4753-E4761.

5. Deng B, et al. Maresin biosynthesis and identification of maresin 2, a new anti-inflammatory and pro-resolving mediator from human macrophages. PLoS One. 2014;9(7):e102362.

6. Serhan CN, et al. Macrophage proresolving mediator maresin 1 stimulates tissue regeneration and controls pain. FASEB J. 2012;26(4):1755-1765.

7. Abdulnour RE, et al. Maresin 1 biosynthesis during platelet-neutrophil interactions is organ-protective. Proc Natl Acad Sci U S A 2014;111(46):16526-16531.

8. Jung TW, Kim HC, Abd El-Aty AM, Jeong JH. Maresin 1 attenuates NAFLD by suppression of endoplasmic reticulum stress via AMPK-SERCA2b pathway. J Biol Chem. 2018;293(11):3981-3988.

9. Laiglesia LM, et al. Maresin 1 mitigates liver steatosis in ob/ob and diet-induced obese mice. Int JObes (Lond). 2018;42(3):572-579.

10. Martínez-Fernández L, et al. Maresin 1 improves insulin sensitivity and attenuates adipose tissue inflammation in ob/ob and diet-induced obese mice. FASEB J. 2017;31(5):2135-2145.

11. Spite M, Clària J, Serhan CN. Resolvins, specialized proresolving lipid mediators, and their potential roles in metabolic diseases. Cell Metab. 2014;19(1):21-36.

12. Han YH, et al. A maresin $1 / \mathrm{ROR} \alpha / 12$-lipoxygenase autoregulatory circuit prevents inflammation and progression of nonalcoholic steatohepatitis. JClin Invest. 2019;129(4):1684-1698.

13. Koyama Y, Brenner DA. Liver inflammation and fibrosis. J Clin Invest. 2017;127(1):55-64.

14. Kang HS, et al. Transcriptional profiling reveals a role for RORalpha in regulating gene expression in obesity-associated inflammation and hepatic steatosis. Physiol Genomics. 2011;43(13):818-828.

15. Kim HJ, et al. Liver-specific deletion of ROR $\alpha$ aggravates diet-induced nonalcoholic steatohepatitis by inducing mitochondrial dysfunction. Sci Rep. 2017;7(1):16041.

16. Lau P, Fitzsimmons RL, Raichur S, Wang SC, Lechtken A, Muscat GE. The orphan nuclear receptor, $\operatorname{ROR} \alpha$, regulates gene expression that controls lipid metabolism: staggerer (SG/SG) mice are resistant to diet-induced obesity. J Biol Chem. 2008;283(26):18411-18421.

17. Han YH, et al. ROR $\alpha$ induces KLF4-mediated M2 polarization in the liver macrophages that protect against nonalcoholic steatohepatitis. Cell Rep. 2017;20(1):124-135.

18. de Urquiza AM, et al. Docosahexaenoic acid, a ligand for the retinoid $\mathrm{X}$ receptor in mouse brain. Science. 2000;290(5499):2140-2144.

19. Chiang N, Serhan CN. Structural elucidation and physiologic functions of specialized pro-resolving mediators and their receptors. Mol Aspects Med. 2017;58:114-129.

20. Krishnamoorthy S, et al. Resolvin D1 binds human phagocytes with evidence for proresolving receptors. Proc Natl Acad Sci U S A. 2010;107(4):1660-1665.

21. Colas RA, et al. Identification and actions of the maresin 1 metabolome in infectious inflammation. J Immunol. 2016;197(11):4444-4452.

22. Rius B, et al. The specialized proresolving lipid mediator maresin 1 protects hepatocytes from lipotoxic and hypoxia-induced endoplasmic reticulum stress. FASEB J. 2017;31(12):5384-5398.

23. Jetten $A M$. Retinoid-related orphan receptors (RORs): critical roles in development, immunity, circadian rhythm, and cellular metabolism. $\mathrm{Nucl}$ Recept Signal. 2009;7:e003.

24. Mamontova A, et al. Severe atherosclerosis and hypoalphalipoproteinemia in the staggerer mouse, a mutant of the nuclear receptor RORalpha. Circulation. 1998;98(24):2738-2743.

25 . Viola JR, et al. Resolving lipid mediators maresin 1 and resolvin D2 prevent atheroprogression in mice. Circ Res. 2016;119(9):1030-1038. 